Technology Spotlight/

\title{
PPAPI: A Program for Groundwater Modeling Tasks in Distributed Parallel Computing Environments
}

Jens Schumacher

Corresponding author: Fluid Domains Inc., 3319 Douglasdale BLVD SE, Calgary, Alberta, Canada;

+1 403370 5150; jschumacher@fluid-domains.com

Kevin Hayley

Groundwater Solutions Pty Ltd., Kensington, Victoria, Australia; kevin.h.hayley@gmail.com

Louis-Charles Boutin

Matrix Solution Inc., Calgary, Alberta, Canada; Iboutin@matrix-solutions.com

Emma White

Groundwater Solutions Pty Ltd., Kensington, Victoria, Australia; kevin.h.hayley@gmail.com

Conflict of interest: None

Key words: Parallel Computing, Calibration, Uncertainty Analysis, Cloud Computing

Article Impact Statement: The software presented in the article helps to run highly parameterized groundwater model calibrations and uncertainty analysis.

Groundwater modeling can involve highly parameterized calibrations, and uncertainty analysis, which both require a significant number of model runs and are time intensive. BeoPEST (Schreuder 2009) and other calibration software is capable of using computational resources distributed across a large number of computers to run parallel calibrations and uncertainty analysis, dramatically reducing run times. Additionally, cloud computing has expanded the computational resources available to groundwater modeling practitioners to almost limitless

This is the author manuscript accepted for publication and has undergone full peer review but has not been through the copyediting, typesetting, pagination and proofreading process, which may lead to differences between this version and the Version of Record. Please cite this article as doi: $10.1111 /$ gwat.12623

This article is protected by copyright. All rights reserved. 
extents. However, the manual distribution of model files, initiation of processes and retrieval of results across a large network of computer resources remained a burdensome task. The software presented here was designed to address the need to automate these tasks.

Model calibration and uncertainty analysis are independent from the time of model initiation to result processing. This independence is termed "embarrassingly parallel". As stated by Fienen and Hunt (2015), the distribution of embarrassingly parallel tasks across distributed computing environments is referred to as High Throughput Computing (HTC), and the open source software HT Condor (HTCondor Team 2014) has been developed to facilitate common HTC tasks. This work presents PPAPI (parallel processes application programming interface), an alternative software solution for HTC groundwater modeling tasks and is based on a platform independent, Python implementation server-client REST (Representational state transfer) architecture using HTTP (Hypertext Transfer Protocol). Testing of PPAPI has been conducted on Microsoft Windows and Linux operating systems and Python versions greater than 2.6.

PPAPI can be used to transfer data, and deploy and control parallel numerical simulations. In addition, it can retrieve simulation results, and perform basic file tasks across computing resources. PPAPI can work within heterogeneous computing environment composed of resources in local area networks (LAN), and external networks such as cloud computing environments. Cloud computing providers, including Amazon, provide remote access to virtually unlimited computing power through their cloud computing infrastructure as a service

This article is protected by copyright. All rights reserved. 
(laaS), enabling computationally intensive analysis that may previously have been unavailable to consultants lacking access to supercomputers or computer clusters. PPAPI, in conjunction with Amazon laaS resources, has been applied to various hydrogeological computing challenges including, highly parameterized inverse problems (Hayley et al. 2014); calibration constrained Monte Carlo Analysis similar to the approach described by Tonkin and Doherty (2009); and the computation of response matrixes to build reduced optimization models based on the approach described by Gorelick (1983). PPAPI consists of two components: a server and a client.

\section{Server component}

The server component of PPAPI runs on all of the resources in the selected distributed computing environment. Initialization of the PPAPI server component at operating system startup can be automated using, for example, Windows task manager or Linux crontab. The server components communicate with a single client via HTTP/HTTPS and manage tasks in response to client requests.

\section{Client component}

Generally, the client component runs on a local computer and communicates with a specified number of servers to manage and distribute tasks (Figure 1). Distributed tasks include initiation of BeoPEST parameter estimation, deletion of BeoPEST files, retrieval of calibration and run

This article is protected by copyright. All rights reserved. 
status, initialization of calibration constrained Monte Carlo analysis including recalibration after parameter projection, and parallel predicative runs with varying parameter sets. A detailed description of the PPAPI software design is available in the user documentation.

Both components of the PPAPI software are available on a public Github repository (https://github.com/PPAPI/ppapi) using the MIT license. The repository contains the source code and user documentation. To illustrate the capabilities of PPAPI, two application examples are provided in the 'test_data' directory. The two examples are explained below.

\section{Example 1: Inverse problem example using BeoPEST}

BeoPEST is a parallel version of the PEST calibration software (Doherty 2010), and can be distributed across computers using TCP/IP protocol. However, BeoPEST does not facilitate distribution and initialization of BeoPEST processes; PPAPI addresses this limitation. PPAPI copies model and PEST files to servers specified by the client component, and starts a defined number of parallel BeoPEST slave processes (Figure 2), on each server and, facilitates restarts of parameter estimation processes (if required) and file cleanup.

Example 2: Subspace Monte Carlo example using PEST

This article is protected by copyright. All rights reserved. 
Calibration constrained Monte Carlo analysis aims to produce multiple realizations of model parameters that fit observed calibration data (Tonkin and Doherty 2009). Part of this process involves running a recalibration simulation for each parameter set produced. PPAPI can be used to run these simulations in parallel.

A calibrated hydraulic conductivity field using PPAPI and BeoPEST is shown on Figure 3 . The synthetic model is described by Moore and Doherty (2005). The corresponding model files and PEST input files are provided for download (http://www.pesthomepage.org/getfiles.php?file=pest uncert.zip). The calibration was conducted using 104 pilot points including regularization and 12 hydraulic head observation points. The calibration method is similar to the approach used by Hayley et al. (2014) where the model runtime of the numerical model, however, was significantly longer due to model size and transient simulation.

PPAPI has been tested and performs well in distributed computing environments with dedicated resources of known and relatively constant computing capacities. On distributed computing environments characterized by high heterogeneity in available computing power, PPAPI could be extended to refine resource allocations by returning information about the CPU (central processing unit), memory and hard drive conditions of a particular computing resource to optimize the distribution of simulations. Furthermore, the client component could be extended to include the dynamic allocation of computing resources, rather than manual

This article is protected by copyright. All rights reserved. 
definition of participating servers for each HTC application. This would be advantageous in distributed computing environments with variable resource allocations, such as the spot market based cloud computing options of Amazon.

\section{References}

Doherty, J. 2010. PEST, Model-independent Parameter Estimation-User Manual, 5thwith slight additions ed. Brisbane, Australia: Watermark Numerical Computing

Fienen, Michael N., and Randall J. Hunt. 2015. High-Throughput Computing Versus HighPerformance Computing for Groundwater Applications. Groundwater 53 (2): 180-84. doi:10.1111/gwat.12320.

Gorelick, Steven M. 1983. A Review of Distributed Parameter Groundwater Management Modeling Methods. Water Resources Research 19 (2): 305-319.

Hayley, Kevin, J. Schumacher, G. J. MacMillan, and L. C. Boutin. 2014. Highly Parameterized Model Calibration with Cloud Computing: An Example of Regional Flow Model Calibration in Northeast Alberta, Canada. Hydrogeology Journal 22 (3): 729-37. doi:10.1007/s10040-014-1110-8.

HTCondor Team. 2014. HTCondor version 8.0 Manual. Technical report, University of Wisconsin- Madison. http://research.cs.wisc.edu/htcondor/ (accessed January 16, 2017).

This article is protected by copyright. All rights reserved. 
Moore, C. and Doherty, J., 2005. The role of the calibration process in reducing model predictive error. Water Resour. Res., 41 (5), W05050.

Schreuder, WA. 2009. Running BeoPEST. In Proceedings of the 1st PEST Conference.

Tonkin, Matthew, and John Doherty. 2009. Calibration-Constrained Monte Carlo Analysis of Highly Parameterized Models Using Subspace Techniques. Water Resources Research 45 (12): W0OB10. doi:10.1029/2007WR006678.

\section{List of Figures}

Figure $1 \quad$ PPAPI server and client communication

Figure 2 PPAPI client output after start of BeoPEST parameter estimation

Figure $3 \quad$ Calibrated hydraulic conductivity field using PPAPI and BeoPEST

This article is protected by copyright. All rights reserved. 


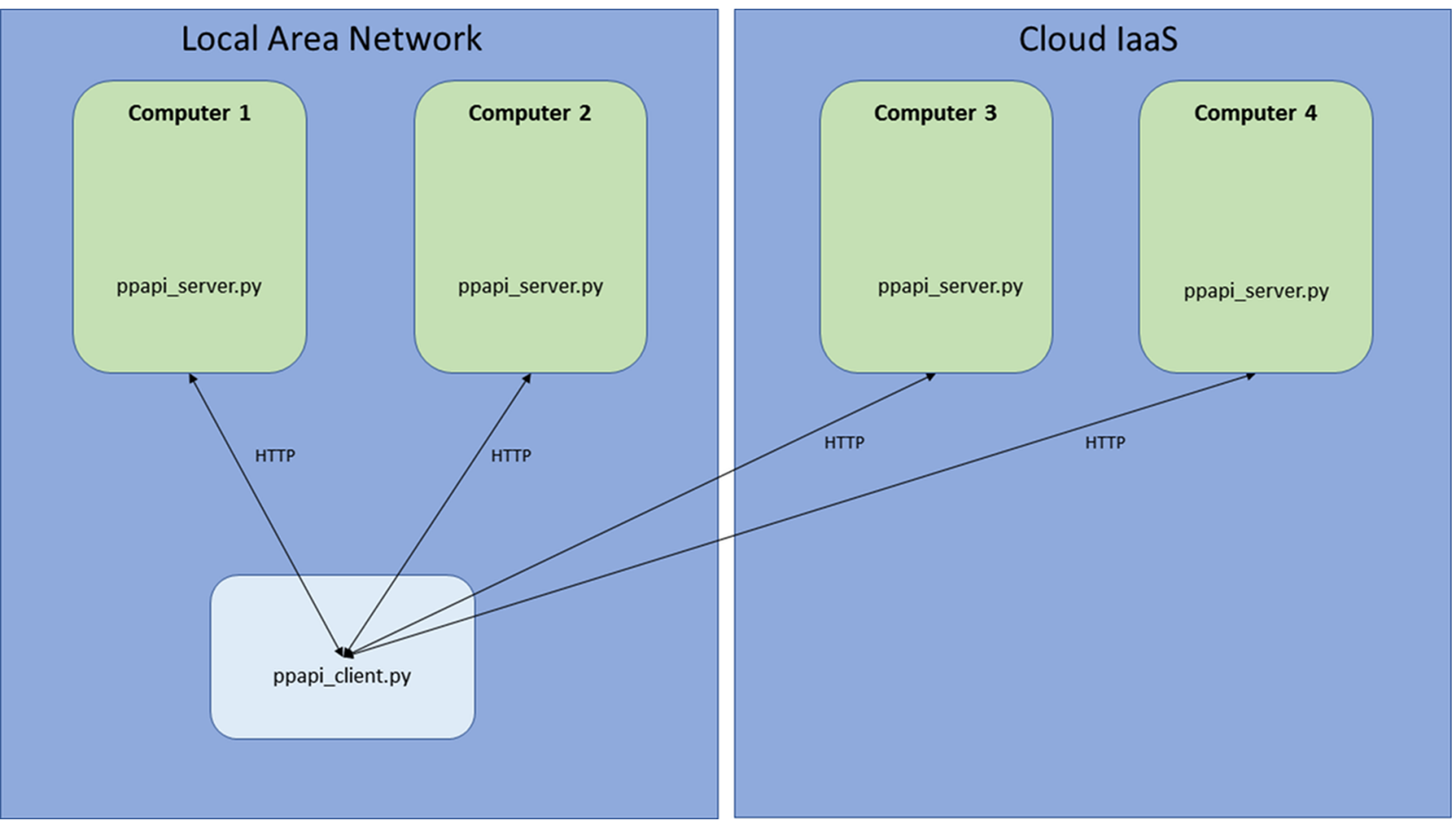

Figure1.tif

This article is protected by copyright. All rights reserved. 
Gi:. C:\WINDOWS \system $32 \backslash \mathrm{cm}$ d.exe

C: \Users\Public>python papi_client.py --case case1 --type beopest --action start -rf calibration_model.zip

f

"hostname": "192.168.1.65:1801",

"runs": [

\{

"run": "case1\} \text { "status", } $\\{\text { "running" }} \end{array}$

\}

\{

"run": "case1\\1",

"status": "running"

\}

start parallel

f

"hostname": "192.168.1.73:1801",

"runs": [

\{

"run": "case1\\2",

"status": "running"

]

\}.

Figure2.tif

This article is protected by copyright. All rights reserved. 


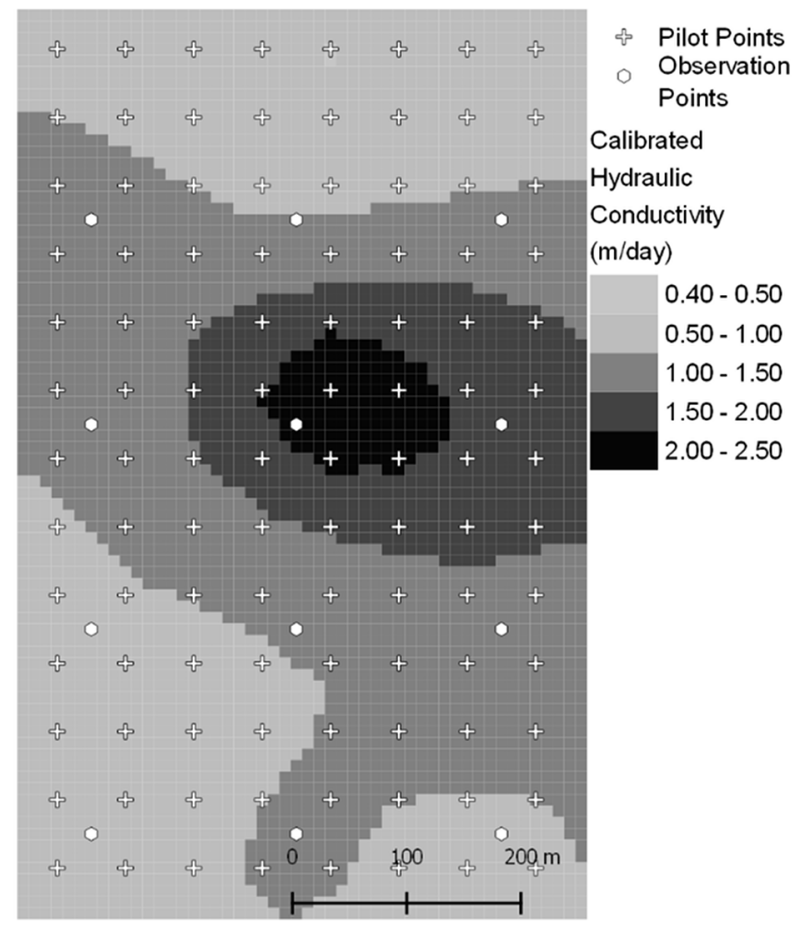

Figure3.tif

This article is protected by copyright. All rights reserved. 


\section{University Library}

\section{- M M N E R VA A gateway to Melbourne's research publications}

Minerva Access is the Institutional Repository of The University of Melbourne

Author/s:

Schumacher, J;Hayley, K;Boutin, L-C;White, E

Title:

PPAPI: A Program for Groundwater Modeling Tasks in Distributed Parallel Computing Environments.

Date:

2018-03

Citation:

Schumacher, J., Hayley, K., Boutin, L. -C. \& White, E. (2018). PPAPI: A Program for Groundwater Modeling Tasks in Distributed Parallel Computing Environments.. Ground Water, 56 (2), pp.248-250. https://doi.org/10.1111/gwat.12623.

Persistent Link:

http://hdl.handle.net/11343/294001 